\title{
A comparative study of intralesional triamcinolone injection in primary chalazion in children and adults
}

\author{
Jagriti Rana1, Santosh Kumar², Mimansaa Agasti ${ }^{3 *}$ \\ ${ }^{\mathbf{1 , 2}}$ Associate Professor, ${ }^{3}$ Senior Resident, Dept. of Ophthalmology, Moti Lal Nehru Medical College, (Affiliated to King George Medical \\ University), India
}

*Corresponding Author: Mimansaa Agasti

Email: obviouslymimansaa@gmail.com

\begin{abstract}
Introduction: A chalazion is a chronic, sterile lipo-granulomatous inflammatory lesion caused due to the blocked sebaceous secretion from the Meibomian and other sebaceous glands. Its painless and harmless, until it becomes an enlarged upper lid chalazion producing mass effect on the cornea leading to blurred vision and astigmatism.

Aim: The aim of our study was to inject $0.1 \mathrm{ml}$ of triamcinolone intralesionally and compare the results in both paediatric and adult age group. We assessed how much time was required for the regression of the lesion and how many patients were in the need for re-injection. We also assessed the need for incision and curettage in such patients.

Materials and Methods: Sixty-seven patients were included in the study, out of which 23 were children and 44 were adults. $0.1 \mathrm{ml}$ of triamcinolone $(40 \mathrm{mg} / \mathrm{dl})$ was injected intralesionally using a 26 gauge needle. The eyelid was everted and the injection was given transconjunctivally into the centre of the lesion. The eye was not patched.

Results: Most lesions resolved within 1 week, the maximum time being 3 weeks in both study populations. Repeat injection was needed in only 1 case in paediatric age group and 15 cases in adult age group. None of our patients developed any adverse effects.

Conclusion: Usually, chalazion is a self-limiting disease and intralesional injection of triamcinolone is a very effective modality for treatment. It can be done under topical anaesthesia, causing much less bleeding and the risk of scarring is almost not there. It is a very quick procedure, performed in a sterilized out-patient setting, requires no special instruments and is very less painful. Its safely tolerated by both children and adults and should be considered as a preferable procedure over incision and curettage.
\end{abstract}

Keywords: Intralesional, Chalazion.

\section{Introduction}

A chalazion is a chronic granulomatous inflammation of the eyelid which is caused by blockage of the openings of the meibomian glands. ${ }^{1}$ It presents as a firm, benign, localized, painless mass in the eyelid, usually affecting the upper eyelid more than the lower eyelid.

There is no sex predilection. ${ }^{2}$ It frequently causes symptoms such as irritation and inflammation. Corneal astigmatism and mechanical ptosis can also result from a large chalazion ${ }^{3}$. Hypermetropia has been also reported. ${ }^{4}$ It can get secondarily infected and result in a painful swelling. ${ }^{5}$

There may be variation in the histopathological appearance but typically the histopathology comprises of a granuloma rich in epithelioid cells, giant cells, lymphocytes, neutrophils and eosinophils. ${ }^{6}$

Certain conditions like seborrhoeic dermatitis, acne rosacea and chronic blepharitis, are factors that predispose to the formation of chalazion. ${ }^{7}$

Chalazion may also be caused by a few viruses. ${ }^{2}$

Most chalazia resolve spontaneously but the rest need some form of treatment. ${ }^{8,9}$

The usual way to treat a chalazion are incision and curettage (I \& C) which is also a standard treatment ${ }^{10}$. Although, this is an extremely effective way to treat a chalazion, but this can be discomforting and painful to a patient. Also, after I \& C, wearing a pad and bandage is required. Intralesional triamcinolone injection is known to hasten the resolution of the chalazion. ${ }^{11}$ This modality is especially useful in children and in chalazia involving the lid margin or close to the punctum and even in multiple chalazia at the same site. ${ }^{12}$

If the lesion does not regress with a single injection of triamcinolone, a second injection is repeated after 6 weeks. ${ }^{10} \mathrm{I} \& \mathrm{C}$ is performed in patients who do not respond to a second injection.

The purpose of this study was to determine the differences of using intralesional triamcinolone acetonide injection for treating primary chalazion in adults and children.

\section{Materials and Methods}

This was a randomised, prospective study conducted at our centre between September 2015 and March 2017. The study was undertaken after ethical clearance was obtained from the Institutional Review Board. Sixty-seven patients were included in the study, out of which 23 were children and 44 were adults. All injections were given by a single ophthalmologist (SR). The inclusion criteria included both male and female patients of all ages (classified later as paediatric group less than 18 years of age and adult group, more than 18 years of age). Patients with primary single chalazion on each lid were chosen.

Exclusion criteria included recurrent chalazion, abnormal surrounding lid tissue and other features indicating malignancy. Also, patients having hypersensitivity to any of the drugs used in the study. Patients having adnexal infection were not included in the study. 
Chalazia which couldn't be palpated were not included in the study. Informed consent was obtained from either the patient, for patients more than 18 years of age or the patients' guardian for patients under 18 years of age.

\section{Technique of Giving Triamcinolone Acetonide Injection}

Proparacaine $0.5 \%$ eye drops were used to anaesthetize the involved eye before injecting triamcinolone. $0.1 \mathrm{ml}$ of triamcinolone $(40 \mathrm{mg} / \mathrm{dl})$ was injected intralesionally using a 26 gauge needle. The eyelid was everted and the injection was given transconjunctivally into the centre of the lesion. The eye was not patched. The injection amount was kept same in both adults and children. After the injection, chloramphenicol eye ointment was given 3 times per day for one week. The patients were called for follow up after one week and reviewed weekly. In case there was incomplete resolution after 3 weeks of injection, the injection was repeated.

\section{Statistical Analysis}

The patients' data was analysed after dividing them into two groups; the paediatric group, which comprised of patients less than 18 years of age and adult group, which comprised of patients more than 18 years of age.

Age, gender, laterality, the time of resolution of the chalazion and the need for a repeat injection, if any, were analysed for both the adult and paediatric age groups using the Mann Whitney $U$ test. Statistical significance was defined as $\mathrm{p}<0.05$.

\section{Results}

Sixty seven patients included in the study out of which 23 were children and 44 were adults. All patients were of Indian origin. No complications were observed in both groups. Table 1 shows the comparative data between adult and paediatric population after chalazion injection.

Table 1: Comparison between the paediatric and adult data after chalazion injection

\begin{tabular}{|l|l|c|c|c|c|}
\hline \multicolumn{2}{|c|}{ Group } & N & Mean & $\begin{array}{c}\text { Standard } \\
\text { deviation }\end{array}$ & p value \\
\hline Age (years) & Paediatric & 23 & 11.52 & 3.489 & 0.0001 \\
\cline { 2 - 6 } & Adults & 44 & 27.39 & 7.711 & \\
\hline $\begin{array}{l}\text { Time for } \\
\text { resolution } \\
\text { (weeks) }\end{array}$ & Paediatric & 23 & 1.30 & 0.703 & 0.198 \\
\cline { 2 - 5 } & Adults & 24 & 1.55 & 0.730 & \\
\hline
\end{tabular}

Table 2:

\begin{tabular}{|l|c|c|}
\hline S. No. & $\begin{array}{c}\text { Paediatric group } \\
\text { (in years) }\end{array}$ & Time for resolution(in weeks) \\
\hline 1. & 17 & 1 \\
\hline 2. & 13 & 1 \\
\hline 3. & 6 & 1 \\
\hline 4. & 8 & 1 \\
\hline 5. & 15 & 1 \\
\hline 6. & 13 & 1 \\
\hline 7. & 13 & 1 \\
\hline 8. & 7 & 1 \\
\hline
\end{tabular}

\begin{tabular}{|l|c|l|}
\hline 9. & 9 & 1 \\
\hline 10. & 10 & 1 \\
\hline 11. & 17 & 3 \\
\hline 12. & 18 & 3 \\
\hline 13. & 7 & 1 \\
\hline 14. & 12 & 1 \\
\hline 15. & 12 & 1 \\
\hline 16. & 9 & 1 \\
\hline 17. & 15 & 1 \\
\hline 18. & 10 & 1 \\
\hline 19. & 6 & 1 \\
\hline 20. & 13 & 2 \\
\hline 21. & 12 & 1 \\
\hline 22. & 12 & 3 \\
\hline 23. & 11 & 1 \\
\hline
\end{tabular}

Repeat injection was given in only 1 case in paediatric age group

Table 3:

\begin{tabular}{|l|c|c|}
\hline S. No. & $\begin{array}{c}\text { Adult group (in } \\
\text { years) }\end{array}$ & $\begin{array}{c}\text { Time for resolution (in } \\
\text { weeks) }\end{array}$ \\
\hline 1. & 21 & 1 \\
\hline 2. & 40 & 2 \\
\hline 3. & 26 & 1 \\
\hline 4. & 19 & 1 \\
\hline 5. & 39 & 2 \\
\hline 6. & 21 & 1 \\
\hline 7. & 20 & 1 \\
\hline 8. & 27 & 1 \\
\hline 9. & 19 & 2 \\
\hline 10. & 27 & 2 \\
\hline 11. & 36 & 1 \\
\hline 12. & 26 & 2 \\
\hline 13. & 21 & 1 \\
\hline 14. & 28 & 1 \\
\hline 15. & 22 & 3 \\
\hline 16. & 24 & 1 \\
\hline 17. & 35 & 3 \\
\hline 18. & 36 & 3 \\
\hline 19. & 42 & 1 \\
\hline 20. & 32 & 1 \\
\hline 21. & 21 & 1 \\
\hline 22. & 33 & 2 \\
\hline 23. & 19 & 1 \\
\hline 24. & 36 & 3 \\
\hline 25. & 21 & 2 \\
\hline 26. & 26 & 2 \\
\hline 27. & 35 & 1 \\
\hline 28. & 25 & 2 \\
\hline 29. & 22 & 1 \\
\hline 30. & 25 & 1 \\
\hline 31. & 40 & 1 \\
\hline 32. & 27 & 1 \\
\hline 33. & 40 & 1 \\
\hline 34. & 20 & 3 \\
\hline 35. & 43 & 2 \\
\hline 36. & 40 & 2 \\
\hline 37. & 20 & 1 \\
\hline 38. & 26 & \\
\hline 39. & 20 & 2 \\
\hline & & 2 \\
\hline .5 & 25 & 2 \\
\hline
\end{tabular}




\begin{tabular}{|l|l|l|}
\hline 40. & 19 & 1 \\
\hline 41. & 20 & 1 \\
\hline 42. & 21 & 1 \\
\hline 43. & 22 & 1 \\
\hline 44. & 23 & 2 \\
\hline
\end{tabular}

Repeat injection was given in 15 cases out of which 1 had to undergo incision \& curettage later in adult age group.

Fig. 1a and $1 \mathrm{~b}$ are the pre injection and post injection pics of a young boy of 10 years from the paediatric age group where as Fig. $2 \mathrm{a}$ and $2 \mathrm{~b}$ depict the pre and post injection pics of a young girl of 22 years from the adult age group which was repeated after 3 weeks.

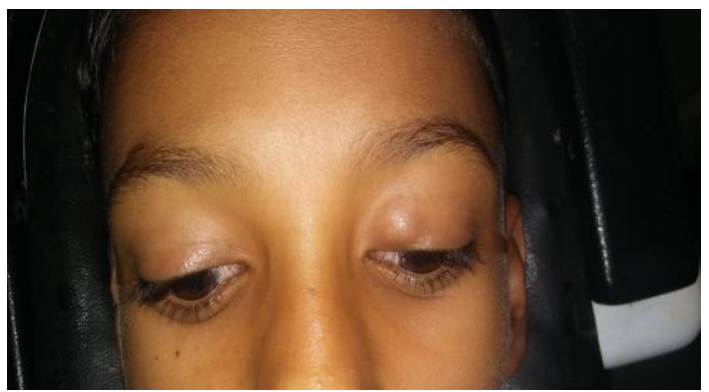

Fig. 1a: Pre-injection picture of a $10 \mathrm{yr}$ kid in paediatric age group

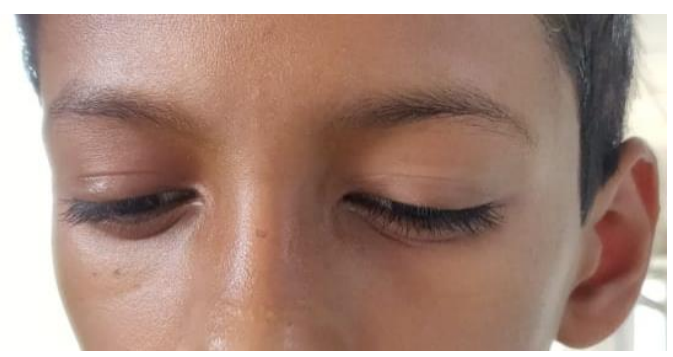

Fig. 1b: Post-injection picture of Intralesional TA in the same kid

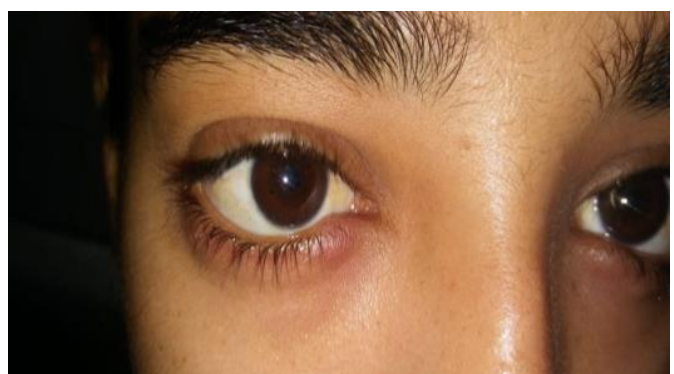

Fig. 2a: Pre-injection picture of a young girl in the adult age group

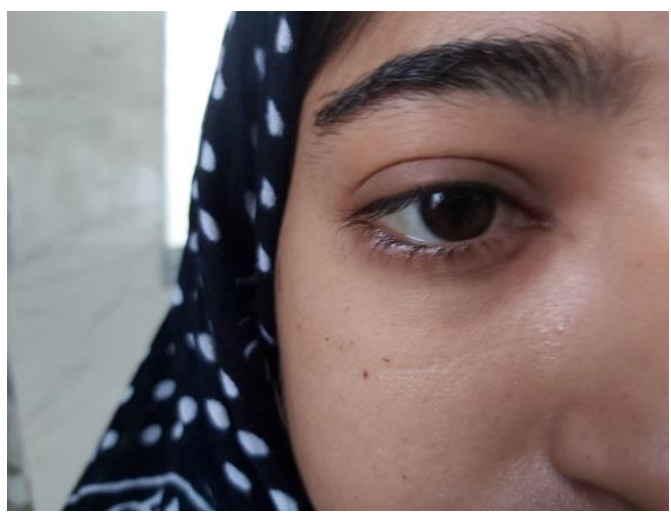

Fig. 2b: Post-injection picture of Intralesional TA in the same girl

\section{Discussion}

Usually, chalazion is a self-limiting disease which occurs across all ages ${ }^{13}$ though more common in the younger age group. Intralesional injection of triamcinolone is known to be a very effective modality treating chalazion. ${ }^{14}$ Although injections need to be repeated before complete chalazion resolution is achieved. ${ }^{15,16}$

An advantage of the transconjunctival route of intralesional triamcinolone over subcutaneous administration of TA is that with the transconjunctival route, the risk of localized skin depigmentation is minimized if it is caused by spillage of the drug around the injection site.

Very important advantage of intralesional triamcinolone is that it can be done under topical anaesthesia, causing much less bleeding and the risk of scarring is almost not there. This is especially useful in children when the chalazion is close to the lacrimal punctum, in cases of multiple chalazia or in a marginal chalazion.

Also it is a very quick procedure, can be performed in a sterilized out-patient setting, requires no special instruments and is very less painful if compared to an anaesthetic injection. The patient can even go back without a patch and so is also preferred in office going adults. This is a useful technique, even in multiple chalazia.

The potential complications which can result from the procedure are elevated intraocular pressure, ${ }^{17}$ and yellowish deposition at the injection site.

But, the most serious adverse effects of intralesional steroid injection is retinal and choroidal vascular occlusion. ${ }^{18}$

In our study, none of the patients reported any adverse effect. The mean time taken for the resolution in the paediatric age group was 9.13 days. Only one case of the paediatric age group was given repeat injection after 3 weeks. One reason could be that it was a very nodular chalazion. Rest all resolved within 1-2 weeks. Two paediatric patients resolved after 3 weeks.

In adults, 42 eyes resolved within 3 weeks and 2 eyes required additional injection after 3 weeks. The mean time taken for resolution in the adult age group is 10.81 days. 
Only one adult required incision and curettage after 3 weeks.

All patients showed compliance with the injections and none required infiltration by a local anaesthetic. Topical anaesthesia seemed to work for all. No eye pad was given post injections and they could resume their day-to-day activities without hindrance of any kind.

The potential limitations of our study was it's small sample size and a control group to compare with other forms of chalazion treatment.

We used a low dosage of injection $(0.1 \mathrm{ml})$ which worked for chalazion of all sizes. Perhaps, increasing the dose for a larger chalazion would have expedited it's resolution but therein lies another lacunae of our study that we didn't correlate the dosage with the size of the chalazion.

All our patients found the injection convenient and we can safely say that our study has effectively proven that intralesional injection as a treatment modality for chalazion was extremely effective in both adults and children. Also, no significant problems occur with the injection and it is safely tolerated by both adults and children and the resolution occurs even with a low dose.

\section{Acknowledgement}

The authors would like to acknowledge each and every patient of the study for having faith in us to allow us the treatment of our choice.

\section{Conflict of Interest: None.}

\section{References}

1. Dua HS ND. Non surgical therapy of chalazion. Am J Ophthalmol 1982;94 (3):424-5.

2. Mansour AM, Chan CC, Crawford MA, Tabbarah ZA, Shen D, Haddad WF, Salti I, Ghazi, NG. Virus induced chalazion. Eye (Lond). 2006;20(2):242-6.

3. Rubin ML Milder B. The fine art of prescribing glasses. Florida Tridd, 1979:98.

4. Santa Cruz CS, Culotta T, Cohen EJ, Rapuano CJ. Chalazion induced hyperopia as a cause of decreased vision. Ophthalmic Surg Lasers 1997;28(8):683-4.
5. Alan H Brigs. The treatment and complications of chalazia. $\mathrm{Br}$ J Ophthalmol 10.1136/bjo 20.2.68.69-73.

6. Pernick N. Eyelid: chalazion of eyelid. Pathology outlines.com website:

https://www.pathologyoutlines.com/topic/eyechalazion.html

7. Suzuki T. Investig Ophthalmol Visual Sci 2018;59:DES94DES101.doi 10.1167/iovs.17-23345

8. Palva J, Pohjanpetto Pos E. J. Intralesional corticosteroid injection for the treatment of chalazia. Acta Ophthalmol (Copenh). 1983;61(5):933-7.

9. Pizzarello LD, Jakobiec FA, Hofeldt AJ, Podelsky MM., Silvers DN. : Intralesional corticosteroid therapy of chalazia. Am J Ophthalmol 1978;85(6):818-21.

10. Goawalla A, Lee V.: A Prospective randomised treatment study comparing three treatment options for chalazia, triamcinolone acetonide injections, incision and curettage and treatment with hot compresses. Clin Exp Ophthalmol. 2007;35(8):706-12.

11. Mustafa TA, Oriafage IH. Three methods of treatment of chalazia in children. Saudi Med J 2001;22(11):968-72.

12. Khurana AK, Ahluwalia BK, Rajan C. Chalazion therapy. Intralesional steroids versus incision and curettage. Acta Ophthalmol (Copenh) 1988;66(3):352-4.

13. Hospital Episode Statistics Department of Health. England 2002-03. (Hospitalization Statistics for Chalazion).

14. Ahmad S, Baig MA, Khan MA, Khan IU, Janjua TA. Intralesional corticosteroid injection vs surgical treatment of chalazia in pigmented patients. J Coll Physicians Surg Pak 2006;16(1):42-4.

15. Cottrell DG, Bosanquet RC, Fawcett IM. Chalazions: The frequency of spontaneous resolution. Br Med J (Clin Res Ed) 1983;26;287(6405):1595.

16. Watson AP, Rustin DJ. Treatment of chalazions with injection steroid suspension. Br J Ophthalmol 1984;68(11):833-5.

17. Lee. Y J, Park CY, Wos KI. Ocular hypertensive response to topical dexamethasone ointment in children. Korean $J$ Ophthalmol 2006;20(3):166-70.

18. Thomas EL, Laborde RP. Retinal and choroidal vascular occlusion following intralesional corticosteroid injection of a chalazion. Ophthalmol 1986;93(3):405-7.

How to cite this article: Rana J, Kumar S, Agasti M. A comparative study of intralesional triamcinolone injection in primary chalazion in children and adults. Int $\mathrm{J}$ Ocul Oncol Oculoplasty 2019;5(2):61-4. 